\title{
Die WLB bildet aus!
}

\section{Die bibliothekarische Ausbildung}

Wie alle großen wissenschaftlichen Bibliotheken in Baden-Württemberg beteiligt sich auch die WLB an der Ausbildung des bibliothekarischen Nachwuchses. Sie ist Ausbildungsbibliothek für die Laufbahnen des mittleren, gehobenen und höheren Bibliotheksdienstes.

Die bibliothekarische Ausbildung hat eine lange Tradition an der WLB. Als Landesbibliothek übernimmt sie auch hier koordinierende Aufgaben für die Region. Das Ministerium für Wissenschaft und Kunst ernannte sie zum 1.10.1984 zur Ausbildungsbehörde für den gehobenen Dienst an wissenschaftlichen Bibliotheken und Dokumentationseinrichtungen. Diese Funktion übte sie bis Ende der neunziger Jahre aus. Der letzte Zulassungsjahrgang der BibliotheksinspektoranwärterInnen beendete im September 1997 seine Ausbildung.

Inzwischen haben sich die Ausbildungswege verändert. Die verwaltungsinterne Ausbildung für den mittleren und den gehobenen Bibliotheksdienst gehört auch in Baden-Württemberg der Vergangenheit an. Mitte der neunziger Jahre wurde die Ausbildung im Beamtenverhältnis durch ein Studium an der Fachhochschule für Bibliothekswesen in Stuttgart abgelöst. Heute bietet die Stuttgarter Hochschule der Medien (HdM) im Studiengang "Informationswissenschaften" zwei Schwerpunkte an: „Bibliotheks-, Kultur- und Bildungsmanagement" und "Daten- und Informationsmanagement".

Für den mittleren Bibliotheksdienst wurde bis zum Frühjahr 2006 verwaltungsintern ausgebildet. Am 1. September begannen die ersten Auszubildenden für den Beruf „Fachangestellter für Medienund Informationsdienste, Fachrichtung Bibliothek (FaMI)" ihre Ausbildung an wissenschaftlichen Bibliotheken.

So auch an der WLB. Und nicht nur unsere ersten Auszubildenden erlebten viel Neues mit uns, wir auch mit der neuen Ausbildung. Es handelt sich um eine dreijährige Ausbildung, die für Abiturienten bzw. Absolventen der Fachhochschulreife um ein Jahr verkürzt werden kann. Im Gegensatz zu vielen anderen Ausbildungsgängen findet der Unterricht an der Berufsschule nicht ein- oder zweimal wöchentlich, sondern blockweise statt. Alle Auszubildenden in Baden-Württemberg besuchen die FaMI-Fachklassen der Hermann-GundertSchule in Calw.

Wie gestalten wir die Ausbildung der angehenden FaMls? Nach einem Einführungskurs, in welchem die Azubis an unser Haus und damit auch an den innen meist unbekannten Bibliothekstyp "wissenschaftliche Bibliothek" herangeführt werden, beginnt der Durchgang durch die Abteilungen. Unser nach den Vorgaben des "Ausbildungsrahmenplans Baden-Württemberg" erstellter Ausbildungsplan beginnt mit den großen Benutzungsbereichen Leihstelle/Magazin und Lesesaal. Danach folgen die einzelnen Arbeitsgebiete der Medienbearbeitung, die Fernleihe und die Information.

Die hier genannten bibliothekarischen Betriebsabteilungen sind auch in den Ausbildungsplänen der folgenden Ausbildungsjahre enthalten. Dazu kommen die bedeutenden Sammlungen unserer Bibliothek, die Bearbeitung kleiner Projekte - z.B. die Erstellung eines Ausbildungs-Wikis für die nächsten Auszubildenden - und Praktika in anderen Bibliotheken und Informationseinrichtungen. Zu den Standardpraktika gehören einwöchige Praktika in der Stadtbibliothek, im Stadtarchiv und in der UB Stuttgart. Absolventen der dreijährigen Ausbildung haben im dritten Ausbildungsjahr auch die Möglichkeit, Informations- und Dokumentations-Einrichtungen kennenzulernen, z.B. die Dokumentation des SWR in Stuttgart oder die Tumordokumentation am Diakonie-Klinikum in Stuttgart.

Informationsbesuche in anderen Einrichtungen runden die Ausbildung ab.

Für Studierende der HdM bietet die WLB Praktikumsplätze an, sowohl für das Kurzpraktikum nach dem ersten Studiensemester als auch für 
das sechsmonatige Praxissemester, welches im fünften Studiensemester stattfindet.

Das Kurzpraktikum stellt für die meisten Studierenden die erste bibliothekarische Arbeitserfahrung dar. Dementsprechend wird es organisiert, die Studierenden sollen Einblicke in unterschiedliche Arbeitsbereiche erhalten, aber auch eine Arbeitsaufgabe näher kennenlernen. In den ersten Jahren war dies die Bearbeitung von Reklamationen in der Pflichtstelle. Derzeit führen die Kurzpraktikantlnnen einfachere Erschließungsarbeiten im Bereich der Nachlasserschließung durch.

Einen sehr viel größeren Raum nimmt die Projektarbeit im Praxissemester ein. Nach dem einführenden dreimonatigen Hausdurchgang bearbeiten die Studierenden allein oder auch zu zweit eine Aufgabenstellung für die Bibliothek. Sie wurde zumeist von der WLB vorgeschlagen. Es gab aber auch Projektthemen, die von den Studierenden eingebracht wurden.

Aufgabenstellungen für die Projektarbeiten kommen aus unterschiedlichen Bereichen des Hauses. Dies verdeutlicht die folgende Aufstellung der Projektthemen aus den Jahren 2010 - 2017:

- Erstellung eines Online-Tutorials zur Zeitungsnutzung,

- Dokumentation der Bucheinbände der Einbandsammlung Gotthilf Kurz,

- Entwicklung eines Konzepts für das Schließsystem im Erweiterungsbau der Landesbibliothek,

- Evaluation der Benutzungsmodalitäten der Musiksammlung im Kontext der musikbibliothekarischen Gesamtsituation in Stuttgart,

- Erschließung eines Teils des sehr umfangreichen Nachlasses des Komponisten und Organisten Hans Georg Bertram (1936-2013),

- Vorbereitung, Durchführung und Auswertung einer Benutzerumfrage zum Hauptlesesaal.

Der Vollständigkeit halber sei noch erwähnt, dass zwar die meisten, aber nicht alle PraktikantInnen an der Stuttgarter Hochschule der Medien studieren. Auch eine Studentin aus Köln absolvierte bei uns ihr Praxissemester und im kommenden Wintersemester 2019/20 wird eine Studentin aus Leipzig ihr Praxissemester in der WLB ableisten.
Für den höheren Bibliotheksdienst wird in BadenWürttemberg noch verwaltungsintern ausgebildet. Als Ausbildungsbehörde übernimmt die WLB hier koordinierende Aufgaben. Jeweils zum 1. Oktober werden - in der Regel fünf - Bibliotheksreferendarlnnen eingestellt. Diese verbringen ihr erstes, praktisches Jahr an einer der Universitäts- oder Landesbibliotheken, das zweite, theoretische Jahr absolvieren sie an der Bayerischen Bibliotheksakademie in München. Im praktischen Jahr sollen sie ihre Ausbildungsbibliothek umfassend kennenlernen. Dies beinhaltet die Arbeitsabläufe in den bibliothekarischen Betriebsabteilungen ebenso wie Verwaltung und Management, aber auch ein intensives Kennenlernen der Fachreferatsarbeit durch Learning by Doing. An der WLB wurden - ihrem geisteswissenschaftlichen Schwerpunkt entsprechend - überwiegend AbsolventInnen geisteswissenschaftlicher Studiengänge als Referendarlnnen eingestellt.

Die Bearbeitung eines größeren Projektes ist auch bei der Referendarausbildung wichtig. Beispielhaft sei hier nur die Erstellung zweier Tutorien zur Literatur- und Bildrecherche im Fach Kunstgeschichte erwähnt.

Praktika an anderen Bibliotheken und Informationsbesuche finden auch im Referendariat statt. Sie werden von den Referendarlnnen ihren fachlichen Schwerpunkten entsprechend in Absprache mit der Ausbildungsleitung organisiert.

Und: Die WLB bietet auch für ReferendarInnen und Auszubildende anderer Bibliotheken Praktika meist einwöchig, aber auch mehrwöchig - an. Aber auch Schülerinnen und Schüler von Gymnasien und Realschulen erhalten bei uns - im Rahmen von BOGY und BORS - die Gelegenheit zur Berufserkundung und damit möglicherweise einen Anhaltspunkt für ihre spätere berufliche Orientierung. Denn die Bibliothek bietet auf unterschiedlichen Qualifikationsstufen interessante Arbeitsplätze an.

Hendrikje Kilian-Schnatterer 